\title{
DNA Light-strand Preferential Recognition of Human Mitochondria Transcription Termination Factor mTERF
}

\author{
Sang-Chul Nam ${ }^{\dagger}$ and Changwon Kang* \\ Department of Biological Sciences, Korea Advanced Institute of Science and Technology, Daejeon 305-701, Korea
}

Received 18 July 2005, Accepted 9 September 2005

\begin{abstract}
Transcription termination of the human mitochondrial genome requires specific binding to termination factor mTERF. In this study, mTERF was produced in $E$. coli and purified by two-step chromatography. mTERF-binding DNA sequences were isolated from a pool of randomized sequences by the repeated selection of bound sequences by gel-mobility shift assay and polymerase chain reaction. Sequencing and comparison of the 23 isolated clones revealed a 16-bp consensus sequence of 5'-GTGTGGCA GANCCNGG-3' in the light-strand (underlined residues were absolutely conserved), which nicely matched the genomic 13-bp terminator sequence 5'-TGGCAGAGCCC GG-3'. Moreover, mTERF binding assays of heteroduplex and single-stranded DNAs showed mTERF recognized the light strand in preference to the heavy strand. The preferential binding of mTERF with the light-strand may explain its distinct orientation-dependent termination activity.
\end{abstract}

Keywords: DNA-protein interaction, DNA strand-specific binding, Human mitochondrial transcription, MELAS mutation, Termination, Terminator, Termination factor mTERF

\section{Introduction}

The two strands of human mitochondrial genome DNA are transcribed to polycistronic RNA molecules originating from displacement-loop promoters, and extend through the entire genome (Clayton, 1984). rRNA genes located in the promoter-proximal region of the heavy-strand (H-strand) are

\footnotetext{
Current Address: Department of Molecular and Cellular Biology, Baylor College of Medicine, One Baylor Plaza, Houston, TX 77030, USA
}

*To whom correspondence should be addressed. Tel: 82-42-869-2628; Fax: 82-42-869-2682

E-mail: ckang@kaist.ac.kr transcribed 50-100 times more than distantly located proteincoding genes. Moreover, mitochondrial transcription termination may be required for the establishment of rRNA gene products (Gelfand and Attardi, 1981), and transcripts initiated at the Hstrand promoter may be elongated or terminated at the 3 ' end of the 16S rRNA gene.

An in vitro transcription assay accurately reflecting the in vivo termination process facilitated initial mitochondrial transcription termination characterization (Christianson and Clayton, 1986). A 13-bp sequence (human mitochondrial DNA nucleotides 3237-3249) located within the tRNA ${ }^{\text {Leu(UUR) }}$ gene was identified by mutagenesis to be essentially required for the termination process (Christianson and Clayton, 1988).

Evidence of a titratable factor responsible for termination further suggested that a site-specific DNA-binding protein plays a crucial role in termination (Christianson and Clayton, 1988). Using DNA affinity chromatography, a fraction called mtTERM was found to be associated with footprinting capacity, and this contained two polypeptides of 34 and $31 \mathrm{kDa}$. Moreover, termination-promoting activity appears to reside in the 34-kDa protein (Daga et al., 1993).

Fernandez-Silva et al. (1997) determined the partial sequences of the $34-\mathrm{kDa}$ protein. Using a partial sequence, they synthesized cDNA and named this encoded protein mTERF. In contrast to purified mTERF, recombinant mTERF synthesized in vitro lacks termination-promoting activity in vitro, although its specific DNA-binding activity remains intact. Recently, rat mTERF was shown to be a phosphoprotein, and its termination activity was found to be inhibited by dephosphorylation (Prieto-Martin et al., 2004). In contrast, the recombinant human $\mathrm{mTERF}$ expressed in insect cells was shown to be fully active in its non-phosphorylated form (Asin-Cayuela et al., 2005). Although some controversy exists concerning the need for phosphorylation for the termination activity of human mTERF, both human and rat mTERF exhibit specific DNA-binding activity in the nonphosphorylated form (Prieto-Martin et al., 2004; AsinCayuela et al., 2005).

Understanding the role of mitochondrial transcription 
termination factor has acquired an additional urgency after it was demonstrated that the point mutation $\mathrm{A} 3243 \mathrm{G}$ is associated with mitochondrial encephalomyopathy lactic acidosis syndrome, or MELAS (Goto et al., 1990; Kobayashi et al., 1990). This mutation dramatically reduces the binding of purified mtTERM and MTERF, and the termination efficiency of mtTERM (Hess et al., 1991; Chomyn et al., 1992). The DNA-binding characteristics of mTERF were examined in this study using recombinant mTERF to investigate the mechanism of transcription termination and MELAS disease.

\section{Materials and Methods}

Construction of an expression vector for mTERF A cDNA clone of the mitochondrial form of mTERF was obtained by PCR using a $\lambda$ Zap cDNA library from HeLa cells (Stratagene, La Jolla, USA). Primers were designed based on Genebank accession number Y09615. The forward primer was 5'-CGGGATCCTTTGG TGTGAAGTGTCATAATACAGACAG-3' and the reverse primer 5'-TGGCAAGCTTAGGCAAATCTGCTTAACTTTTTCAATTTA-3' (BamHI and HindIII restriction sites are underlined). A BamHIHindIII fragment of the polymerase chain reaction (PCR) products was inserted into the BamHI and HindIII sites of pGEX-KG (Guan and Dixon, 1991), to produce pGEX-mTERF. The insert sequence was confirmed on both strands by the dideoxynucleotide chain termination method.

Expression and purification of recombinant mTERF $E$. coli BL21/pGEX-mTERF was cultured in LB medium containing $100 \mathrm{mg} / \mathrm{ml}$ ampicillin at $37^{\circ} \mathrm{C}$. Expression was induced at $25^{\circ} \mathrm{C}$ with $0.4 \mathrm{mM}$ isopropyl- $\beta$-thiogalactopyranoside (IPTG) at an $A_{600 \mathrm{~nm}}$ of $\sim 0.4$ for $4 \mathrm{~h}$. Cell pellets harvested by centrifugation were resuspended in TNE buffer ( $20 \mathrm{mM}$ Tris- $\mathrm{HCl}(\mathrm{pH} 8.0), 150 \mathrm{mM}$ $\mathrm{NaCl}, 1 \mathrm{mM}$ EDTA, $1 \mathrm{mM}$ dithiothreitol) supplemented with 0.1 $\mathrm{mM}$ phenylmethylsulfonyl fluoride (PMSF) and 0.1\% Tween-20. After sonication and centrifugation, the supernatant was loaded on a glutathione-agarose column (Sigma-Aldrich, St. Louis, USA). After extensive washing with TNE buffer, glutathione S-transferase (GST) fusion proteins were eluted with buffer (100 mM Tris-HCl (pH 8.0), $150 \mathrm{mM} \mathrm{NaCl}, 10 \mathrm{mM}$ glutathione (reduced form), $1 \mathrm{mM}$ dithiothreitol), and dialyzed in buffer containing $20 \mathrm{mM}$ Tris-HCl (pH 8.0), $150 \mathrm{mM} \mathrm{NaCl}, 5 \%$ glycerol, $1 \mathrm{mM}$ dithiothreitol and $1 \mathrm{mM}$ EDTA. A portion of the fusion protein mixture was digested with thrombin (Sigma-Aldrich, St. Louis, USA), and inactivated by adding $1 \mathrm{mM}$ PMSF. Fractions were passed through a glutathioneagarose column to remove undigested fusion proteins and digested GST fragments.

Thrombin-digested and -undigested recombinants were further purified using phosphocellulose columns. GST-mTERF and mTERF were eluted with $500 \mathrm{mM} \mathrm{NaCl}$, dialyzed in a storage buffer (20 mM Tris-HCl (pH 8.0), $1 \mathrm{mM}$ EDTA, $150 \mathrm{mM} \mathrm{NaCl}, 1 \mathrm{mM}$ dithiothreitol, $5 \%$ glycerol), and stored at $-80^{\circ} \mathrm{C}$. Their concentrations were determined using Bradford reagent (Bio-Rad Laboratories, Hercules, USA).

Binding site selection by electrophoretic mobility shift analysis A pool of 59-bp DNA oligomers with sequence variations at 17 positions were constructed by PCR using a synthetic 59-nt template

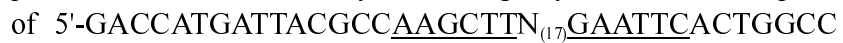
GTCGTTTT-3' (HindIII and EcoRI sites are underlined). The 17-nt region was surrounded by two 21-nt constant sequences that were used as 21-nt forward and reverse primers. PCR was performed using radioactive dNTP for 10 amplification cycles \{denaturation $\left(94^{\circ} \mathrm{C}, 30 \mathrm{~s}\right)$, annealing $\left(55^{\circ} \mathrm{C}, 30 \mathrm{~s}\right)$ and extension $\left.\left(72^{\circ} \mathrm{C}, 30 \mathrm{~s}\right)\right\}$. $\mathrm{PCR}$ products obtained were treated with phenol/chloroform.

PCR products $(0.1 \mu \mathrm{g})$ were incubated with mTERF $(0.1 \mu \mathrm{g})$ at $30^{\circ} \mathrm{C}$ for $30 \mathrm{~min}$ in binding buffer consisting of $20 \mathrm{mM}$ Tris- $\mathrm{HCl}(\mathrm{pH}$ 8.0), $10 \mathrm{mM} \mathrm{MgCl}_{2}, 50 \mathrm{mM} \mathrm{NaCl}, 50 \mu \mathrm{g} / \mathrm{ml}$ poly(dI-dC), $0.1 \mu \mathrm{g} / \mathrm{ml}$ bovine serum albumin, $1 \mathrm{mM}$ dithiothreitol, $0.01 \%$ Tween 20 , and $5 \%$ glycerol. This mixture was subjected to electrophoretic mobility shift assays (Ganguly et al., 2004) using 5\% (w/v) polyacrylamide gel electrophoresis in $0.5 \times$ TBE buffer. Shifted radioactive bands were cut into pieces and the DNA was extracted in $200 \mu \mathrm{l}$ of $0.5 \times \mathrm{TE}$ buffer at $25^{\circ} \mathrm{C}$ for $16 \mathrm{~h}$. The extract was centrifuged at $10,000 \times \mathrm{g}$ for $1 \mathrm{~min}$ and the supernatant was treated with phenol/ chloroform. One $\mu \mathrm{l}$ of the DNA solution was again subjected to PCR using the primers mentioned above and the product DNA so obtained was used for protein binding. PCR/electrophoretic mobility shift processes (Jing et al., 2004) were repeated 15 times. The electrophoretic mobility shift results on each occasion were compared in order to monitor the enrichment of sequences that specifically bound mTERF. Because the selected pools reached the saturation level at the 12th cycle, the PCR products of the 12th cycle were digested with EcoRI and HindIII, and subcloned into the same restriction sites of pUC19 for sequencing.

DNA-binding assays Various DNA probes were tested for binding to mTERF using mobility shift assays. A 28-nt oligomer Wu (5'TGTTAAGATGGCAGAGCCCGGTAATCGC-3') had the same sequence as the L-strand of the human mitochondrial transcription terminator locus MTTER (mitochondrial DNA nucleotides 32293256). A substitution mutant $\mathrm{Mu}$ (5'-TGTTAAGATGGCAGGGCC CGGTAATCGC-3') carried a MELAS mutation A3243G (underlined). Another mutant Du (5'-TGTTAAGATGGCAG-CGGTAATCGC3') carried a 4-nt deletion (dashed). Wl, Ml and Dl had sequences complementary to those of $\mathrm{Wu}, \mathrm{Mu}$ and $\mathrm{Du}$, respectively (u stands for the upper L-strand and 1 for the lower $\mathrm{H}$-strand). Thus, Wu and $\mathrm{Wl}$ oligomers would form a duplex as would $\mathrm{Mu} / \mathrm{Ml}$ and $\mathrm{Du} / \mathrm{Dl}$.

Fifty fmol of probes, individuals or duplexes, were incubated with $100 \mathrm{ng}$ of purified proteins in $20 \mu \mathrm{l}$ of $20 \mathrm{mM}$ Tris- $\mathrm{HCl}(\mathrm{pH}$ 8.0), $50 \mathrm{mM} \mathrm{NaCl}, 10 \mathrm{mM} \mathrm{MgCl}, 1 \mu \mathrm{g}$ of poly(dI-dC), $0.1 \mu \mathrm{g} / \mathrm{ml}$ bovine serum albumin, $1 \mathrm{mM}$ dithiothreitol, $5 \%$ glycerol at $30^{\circ} \mathrm{C}$ for $30 \mathrm{~min}$. Mixtures were then loaded on a native $5 \%$ polyacrylamide gel in $0.5 \times$ TBE buffer, and run as previously described (FernandezSilva et al., 1996). Heat-denatured poly(dI-dC) was used instead of duplex poly(dI-dC) as a nonspecific competitor for single-strand DNA binding assays. 


\section{Results and Discussion}

Production of recombinant $m$ TERF in $E$. coli and its purification The human mitochondrial protein mTERF binds the terminator sequence in the human mitochondrial genome (Daga et al., 1993). A recombinant mTERF produced by the in vitro translation system of rabbit reticulocyte lysate also showed terminator-specific DNA-binding activity, although it failed to render termination (Fernandez-Silva et al., 1997). In this study, a mitochondrial form of mTERF was produced using an $E$. coli expression system to eliminate possible reticulocyte protein contamination and to determine the DNA binding specificity of human mTERF. Using pGEX-KG vector, mTERF lacking mitochondrial signal peptide was obtained fused to an N-terminal GST tag containing a thrombin digestion site (GST-mTERF). When treated with thrombin, mTERF contained two additional residues (GlySer) at its N-terminus.

The mature form of mTERF purified to near homogeneity, showed an apparent molecular mass of $34 \mathrm{kDa}$ by SDSpolyacrylamide gel migration (Fig. 1A), which was consistent with previous estimations for protein produced using reticulocyte lysates (Fernandez-Silva et al., 1997), and with that for protein purified from human mitochondria (Hess et al., 1991; Daga et al., 1993). Although its amino acid sequence predicts $39 \mathrm{kDa}$, mTERF's strong tertiary structure could explain this discrepancy (Fernandez-Silva et al., 1997).

Specific binding of mTERF to terminator DNA In vitro DNA binding tests using the 28-bp probe of $\mathrm{Wu} / \mathrm{Wl}$ carrying the entire human mitochondrial terminator MTTER sequence, were performed by gel mobility shift analysis (Fig. 1B). Both mTERF and GST-mTERF strongly bound to the probe, and the GST fusion did not affect the binding capacity of mTERF. The binding constants of recombinant mTERF and GSTmTERF were similar to that of protein produced by in vitro translation using reticulocyte lysates (Fernandez-Silva et al., 1997).

The DNA-binding specificity of mTERF was tested using competitors by gel mobility shift analysis (Fig. 1C). The retarded complex of mTERF and radioactive $\mathrm{Wu} / \mathrm{Wl}$ probe (lane 2) was completely competed out by a 10-fold molar excess of non-radioactive $\mathrm{Wu} / \mathrm{Wl}$ probe (lane 3 ), but was not by a 10 -fold molar excess of $\mathrm{Du} / \mathrm{Dl}$ probe carrying a 4-bp deletion (lane 5). The complex was also competed away by $80 \%$, by the probe $\mathrm{Mu} / \mathrm{Ml}$ carrying a MELAS mutation (lane 4). These results show that the MELAS mutation substantially decreases mTERF binding to the terminator sequence, but that it does not abolish it, whereas the 4-bp deletion abolishes this binding. Our recombinant mTERF protein had terminator sequence-specific binding activity alone, and did not need mammalian phosphorylation for DNA binding. Moreover, the extra two N-terminal residues or GST tag did not interfere with its binding specificity.

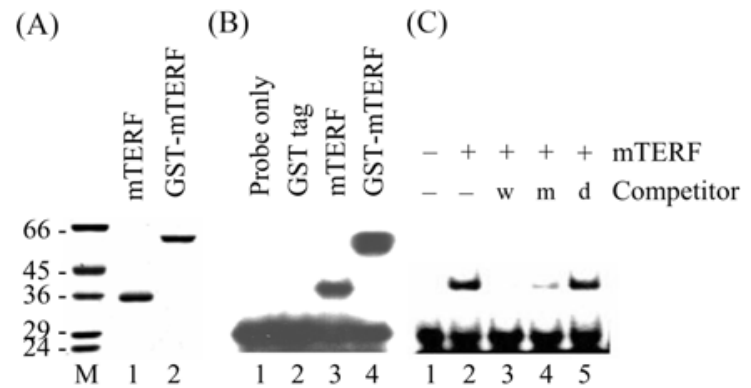

Fig. 1. Preparation and DNA-binding capacity of recombinant mTERF and GST-mTERF. (A) The mitochondrial forms of mTERF without (lane 1) and with a GST tag at the N-terminus (lane 2) were produced in E. coli, purified by two-step chromatography, subjected to $10 \%$ SDS-polyacrylamide gel electrophoresis, and stained with Coomassie brilliant blue. (B) A 28-bp radio-labeled DNA probe $\mathrm{Wu} / \mathrm{Wl}$ (lane 1) was incubated with GST tag (lane 2), mTERF (lane 3), or GST-mTERF (lane 4) for $30 \mathrm{~min}$ at $30^{\circ} \mathrm{C}$ and subjected to gel-mobility shift assays. (C) Radio-labeled probe $\mathrm{Wu} / \mathrm{Wl}$ (lane 1) was incubated with mTERF in the absence (lane 2) and in the presence of a 10-fold excess of unlabeled competitor $\mathrm{Wu} / \mathrm{Wl}(\mathrm{w})$ of the wild type (lane 3), $\mathrm{Mu} / \mathrm{Ml}(\mathrm{m})$ carrying a MELAS mutation (lane 4), or $\mathrm{Du} / \mathrm{Dl}$ (d) carrying a 4-bp-deletion (lane 5).

Selection of mTERF-binding DNA sequences Purified recombinant $\mathrm{MTERF}$ protein was used to select binding sequences from a random pool of double-stranded DNA. A previous mutagenesis study determined a $13 \mathrm{bp}$ sequence (human mitochondrial DNA nucleotides 3237-3249) to be essential for termination (Christianson and Clayton, 1988). Seventeen consecutive positions in the middle of 59-bp DNA oligomers were randomized and subjected to 12 cycles of binding selection and amplification. The resulting, enriched oligomers were then cloned and 23 clones were sequenced (Fig. 2A).

All clones shared a TGGC sequence, and thus were aligned relative to this sequence, which was arbitrarily numbered from 10 to 13 . Based on residue frequencies at each position (Fig. 2B), sequence logos are presented in Fig. 2C. The resulting consensus sequence of $16 \mathrm{bp}$ was derived as GTG TGGCAGANCCNGG (the absolutely conserved positions are underlined) at positions 7 through 22. (NOTE: The sequence logos at positions $1-5$ and 23-25 reflect sequences fixed in the randomized pool and should not be regarded as consensus sequence). This 16-bp consensus sequence fits the 13-bp natural terminator sequence of TGGCAGACCCCGG, except for the first three residues (GTG), which differ from the natural sequence (AGA). Although the 3-bp AGA was not found in the positions 7-9 of the 23 sequences, individual residues $\mathrm{A}, \mathrm{G}$, and $\mathrm{A}$ were found at positions 7,8 and 9, respectively (Fig. 2B). Thus, positions 7-9 may not be essential for DNA binding, and GTG could be preferred. However, the number (23) of mTERF-binding sequences isolated was too small to make this statistically certain. No 
(A)

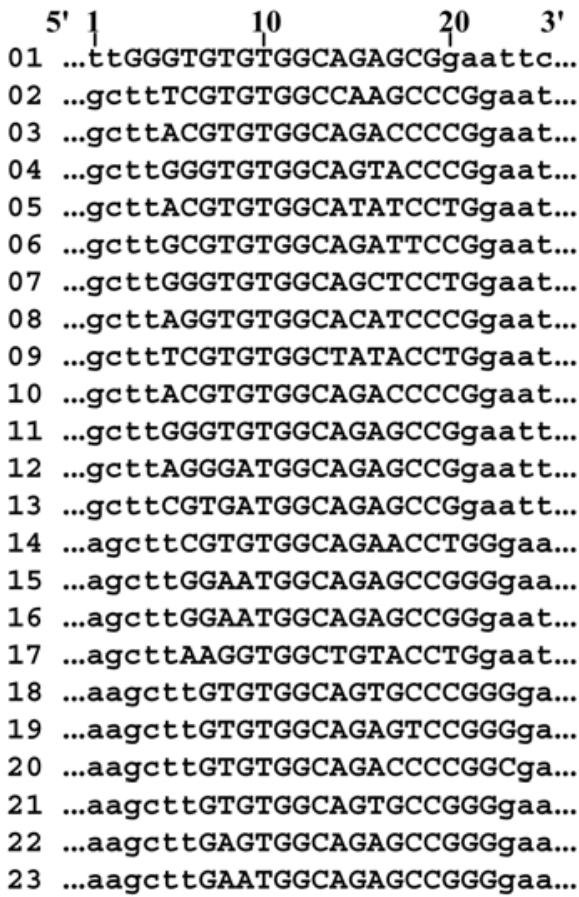

(B)

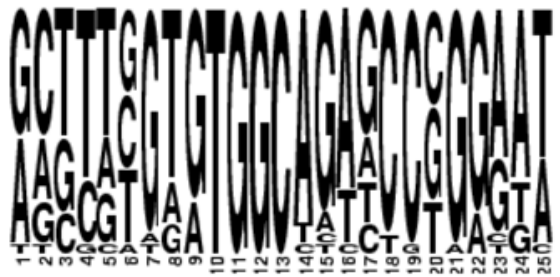

(C)

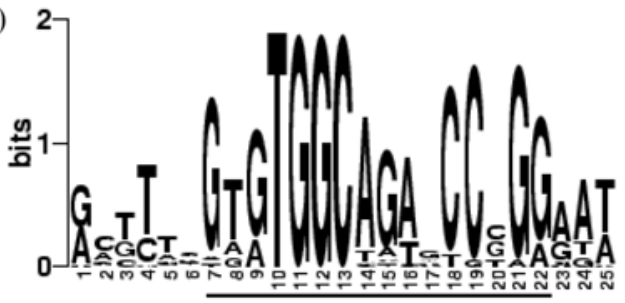

Fig. 2. Selection of mTERF-binding sequences. (A) From a pool of 59-bp DNAs randomized in the middle 17-bp region, 23 clones binding to recombinant mTERF were isolated and sequenced. Sequences are aligned relative to the absolutely conserved TGGC whose positions were numbered 10 through 13 . The randomized region is shown by capital letters and the partial sequences of the fixed regions by lower case. (B) The relative frequencies of individual residues at each position from 1 to 25 are represented as relative character heights. (C) Sequence logos were generated from $23 \mathrm{mTERF}-b i n d i n g$ sequences via http:// www.bio.cam.ac.uk/seqlogo/logo.cgi (Schneider and Stephens, 1990). The information content amount at each position is represented by position height. The consensus sequence region (positions 7-22) is shown by a bar.

other sequences similar to the consensus sequence were found in the human mitochondrial genome.

Position 16 corresponds to the genomic position 3243, which carries an $\mathrm{A} \rightarrow \mathrm{G}$ mutation in MELAS. It was not
(A)

(B)

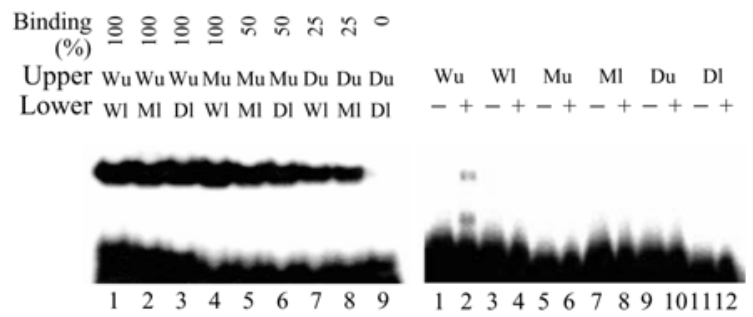

Fig. 3. DNA binding specificity of mTERF. (A) Upper-strand oligomers $\mathrm{Wu}, \mathrm{Mu}$, and $\mathrm{Du}$ were radio-labeled at their $5^{\prime}$ ends and mixed with an equimolar amount of lower-strand oligomers $\mathrm{Wl}, \mathrm{Ml}$ and $\mathrm{Dl}$ in all possible combinations. Each homoduplex or heteroduplex was incubated with recombinant mTERF and subjected to gel-mobility shift assay. Bound complex amounts were measured by phosphoimaging shifted bands and the values shows are relative to those of wild-type duplex $\mathrm{Wu} / \mathrm{Wl}$. Errors were within $10 \%$. (B) Bindings of mTERF to single-stranded DNA, $\mathrm{Wu}, \mathrm{Wl}, \mathrm{Mu}, \mathrm{Ml}, \mathrm{Du}$ or $\mathrm{Dl}$ were assayed individually with $(+)$ or without $(-)$ mTERF and subjected to gel-mobility shift assays.

absolutely conserved in our selection, but preferentially carried the A residue in 17 of 23 clones. Other observed residues were $\mathrm{T}$ in 5 clones and $\mathrm{C}$ in 1 clone, but none of the clones possessed $\mathrm{G}$ (Fig. 2B). Thus, the avoidance of a $\mathrm{G}$ residue in position 16 explains reduced mTERF binding to DNA carrying the A3243G MELAS mutation.

Binding of mTERF to heteroduplex and single-strand DNA We investigated whether the terminator sequence must be present in both strands of DNA to mediate mTERF binding, since the transcription termination sequence is known to bind mTERF. Various heteroduplexes were constructed using the upper-strand (L-strand) oligomers $\mathrm{Wu}, \mathrm{Mu}$, and $\mathrm{Du}$, and the lower-strand (H-strand) oligomers Wl, Ml and Dl, and were tested for mTERF-binding by using gel retardation assays (Fig. 3A).

When both strands carried the 4-bp deletion (Du/Dl), mTERF binding was completely abolished (lane 9). In contrast, the MELAS mutation homoduplex Mu/Ml showed half binding (lane 5) compared with the wild-type duplex $\mathrm{Wu}$ / $\mathrm{Wl}$ (lane 1). These results are consistent with the competition assay results shown above (Fig. 1C), in that the 4-bp deletion abolishes mTERF binding, and that the MELAS mutation reduces it substantially.

When the lower strand of wild-type Wl was replaced by the deletion mutant $\mathrm{Dl}$ in heteroduplexes with $\mathrm{Wu}$, DNA binding was not significantly changed (lane 3 ). In contrast, DNA binding was reduced by three quarters when the upper strand Wu was replaced by Du in heteroduplexes with Wl (lane 7). Also, when the opposite strand carried the MELAS mutation, the effect of the 4-nt deletion in the upper strand was a threequarter reduction in $\mathrm{mTERF}$ binding ( $\mathrm{Du} / \mathrm{Ml}$ in lane 8 had one-quarter of the activity of $\mathrm{Wu} / \mathrm{Ml}$ in lane 2 ), but the effect 
of the 4-nt deletion in the lower strand was only a $50 \%$ reduction in the binding $(\mathrm{Mu} / \mathrm{Dl}$ in lane 6 showed $50 \%$ of the activity of $\mathrm{Mu} / \mathrm{Wl}$ in lane 4). Thus, the binding-interfering effect of the 4-bp deletion was more severe in the upper strand (Du) than in the lower strand (Dl), when the opposite strand was either the wild-type or carried the MELAS mutation.

The effect of the MELAS mutation was also more severe in the upper strand $(\mathrm{Mu})$ than in the lower strand $(\mathrm{Ml})$, when opposite strands carried the 4-base deletion (Dl or Du), since $\mathrm{Mu} / \mathrm{Dl}$ (lane 6) showed $50 \%$ binding activity of $\mathrm{Wu} / \mathrm{Dl}$ (lane 3 ), whereas Du/Ml (lane 8) showed the same activity as Du/ Wl (lane 7). In summary, these results indicate that the upperstrand (L-strand) sequence is more important in mTERF binding than the lower-strand (H-strand) sequence.

Our heteroduplex results led us to examine whether mTERF can bind single-stranded DNA (Fig. 3B). The upper strand $\mathrm{Wu}$ (L-strand of wild-type terminator sequence) alone bound to mTERF (lane 2), whereas the lower strand (Hstrand) Wl did not (lane 4). These results of single strands and heteroduplexes suggest that mTERF binding relies more on the L-strand than the H-strand of the terminator sequence. The other single strands $\mathrm{Mu}, \mathrm{Ml}, \mathrm{Du}$, and Dl did not bind mTERF (lanes 6, 8, 10, and 12, respectively), indicating that the MELAS mutation and 4-bp deletion reduce single stranded DNA binding in addition to duplex DNA binding.

\section{Implications of the preferential recognition of light strand} by mTERF The preference of mTERF for upper L-strand binding of the terminator sequence over the lower H-strand observed in this study, may explain the distinct orientationdependent termination activity of mTERF reported by AsinCayuela et al. (2005). Transcription termination is almost complete when the L-strand is oriented as the upper strand of the terminator, but only partial when the terminator is in the reverse orientation (Asin-Cayuela et al., 2005). Thus, the dependency of the termination activity of MTERF on orientation can be ascribed at least in part to the orientation preference of mTERF binding to the terminator.

Acknowledgments This work was supported by a grant from the Korean Ministry of Science and Technology. CK was a participant to the Brain Korea 21 Project.

\section{References}

Asin-Cayuela, J., Schwend, T., Farge, G. and Gustafsson, C. M. (2005) The human mitochondrial transcription termination factor (mTERF) is fully active in vitro in the nonphosphorylated form. J. Biol. Chem. 280, 25499-25505.

Chomyn, A., Martinuzzi, A., Yoneda, M., Daga, A., Hurko, O., Johns, D., Lai, S. T., Nonaka, I., Angelini, C. and Attardi, G. (1992) MELAS mutation in mtDNA binding site for transcription termination factor causes defects in protein synthesis and in respiration but no change in levels of upstream and downstream mature transcripts. Proc. Natl. Acad.
Sci. USA 89, 4221-4225.

Christianson, T. W. and Clayton, D. A. (1986) In vitro transcription of human mitochondrial DNA: accurate termination requires a region of DNA sequence that can function bidirectionally. Proc. Natl. Acad. Sci. USA 83, 6277-6281.

Christianson, T. W. and Clayton, D. A. (1988) A tridecamer DNA sequence supports human mitochondrial RNA 3'-end formation in vitro. Mol. Cell. Biol. 8, 4502-4509.

Clayton, D. A. (1984) Transcription of the mammalian mitochondrial genome. Annu. Rev. Biochem. 53, 573-594.

Daga, A., Micol, V., Hess, D., Aebersold, R. and Attardi, G. (1993) Molecular characterization of the transcription termination factor from human mitochondria. J. Biol. Chem. 268, 8123-8130.

Fernandez-Silva, P., Martinez-Azorin, F., Micol, V. and Attardi, G. (1997) The human mitochondrial transcription termination factor (mTERF) is a multizipper protein but binds to DNA as a monomer, with evidence pointing to intramolecular leucine zipper interactions. EMBO J. 16, 1066-1079.

Fernandez-Silva, P., Micol, V. and Attardi, G. (1996) Mitochondrial DNA transcription initiation and termination using mitochondrial lysates from cultured human cells. Methods Enzymol. 264, 129-139.

Ganguly, T., Chattoraj, P., Das, M., Chanda P. K., Mandal, N. C., Lee, C. Y. and Sau, S. (2004) A point mutation at the Cterminal half of the repressor of temperate mycobacteriophage L1 affects its binding to the operator DNA. J. Biochem. Mol. Biol. 37, 709-714.

Gelfand, R. and Attardi, G. (1981) Synthesis and turnover of mitochondrial ribonucleic acid in HeLa cells: the mature ribosomal and messenger ribonucleic acid species are metabolically unstable. Mol. Cell. Biol. 1, 497-511.

Goto, Y., Nonaka, I. and Horai, S. (1990) A mutation in the tRNA(Leu)(UUR) gene associated with the MELAS subgroup of mitochondrial encephalomyopathies. Nature 348, 651-653.

Guan, K. L. and Dixon, J. E. (1991) Eukaryotic proteins expressed in Escherichia coli: an improved thrombin cleavage and purification procedure of fusion proteins with glutathione S-transferase. Anal. Biochem. 192, 262-267.

Hess, J. F., Parisi, M. A., Bennett, J. L. and Clayton, D. A. (1991) Impairment of mitochondrial transcription termination by a point mutation associated with the MELAS subgroup of mitochondrial encephalomyopathies. Nature 351, 236-239.

Jin, Z., Liu, Y., Dong, M., Hu, S. and Huang, S. (2004) Identification of the DNA binding element of the human ZNF333 protein. J. Biochem. Mol. Biol. 37, 663-670.

Kobayashi, Y., Momoi, M. Y., Tominaga, K., Momoi, T., Nihei, K., Yanagisawa, M., Kagawa, Y. and Ohta, S. (1990) A point mutation in the mitochondrial tRNA(Leu)(UUR) gene in MELAS (mitochondrial myopathy, encephalopathy, lactic acidosis and stroke-like episodes). Biochem. Biophys. Res. Commun. 173, 816-822.

Prieto-Martin, A., Montoya, J. and Martinez-Azorin, F. (2004) Phosphorylation of rat mitochondrial transcription termination factor (mTERF) is required for transcription termination but not for binding to DNA. Nucleic Acids Res. 32, 2059-2068.

Schneider, T. D. and Stephens, R. M. (1990) Sequence logos: a new way to display consensus sequences. Nucleic Acids Res. 18, 6097-6100. 